\title{
PERDA DE CARGA LOCALIZADA EM GOTEJADORES INTEGRADOS EM TUBOS DE POLIETILENO
}

\section{ANTHONY W. A. GOMES ${ }^{1}$, JOSÉ A. FRIZZONE ${ }^{2}$, OSVALDO RETTORE NETO ${ }^{3}$, JARBAS H. DE MIRANDA ${ }^{4}$}

RESUMO: Este trabalho foi desenvolvido para analisar as perdas de carga localizadas em gotejadores coaxiais integrados em tubos de polietileno. A perda de carga para diferentes vazões foi determinada em quatro modelos de tubos gotejadores, com sete repetições. Para cada vazão, a perda de carga localizada foi calculada pela diferença entre a perda de carga no tubo com emissor e a perda de carga contínua no tubo uniforme, estimada pela equação de Darcy-Weisbach. Aproximações matemáticas foram sugeridas para calcular a perda de carga com base no coeficiente de carga cinética $(\mathrm{K})$ e em um valor constante de comprimento equivalente (Le). A desconsideração da perda de carga localizada levou à superestimativa do comprimento máximo da linha lateral de até $25,7 \%$, para os gotejadores autocompensados, e de 9,5\%, para os não autocompensados. $\mathrm{O}$ cálculo da perda de carga localizada, utilizando o modelo potencial para estimar o valor de $\mathrm{K}$ em função do índice de obstrução, mostrou bons resultados, comparáveis àqueles obtidos com o valor de $\mathrm{K}$ ajustado pelos dados observados em laboratório, o que resultou em pequenas variações na estimativa do comprimento máximo da linha lateral por esses dois procedimentos.

PALAVRAS-CHAVE: irrigação por gotejamento, coeficiente de carga cinética, perda de carga localizada, índice de obstrução, comprimento equivalente.

\section{LOCAL HEAD LOSSES FOR INTEGRATED DRIPPERS IN POLYETHYLENE PIPES}

\begin{abstract}
This work was developed to determine localized head losses in coaxial emitter integrated in polyethylene pipes. The localized head losses, for different flows, were obtained in four emitters' model, with seven replications. The amount of local losses caused by the emitter was calculated by the difference between the total measured head losses of the segment and the corresponding friction losses evaluated by the Darcy-Weisbach equation. Mathematical approaches were suggested to calculate the head loss using the kinetic load coefficient $(\mathrm{K})$ and a constant value of equivalent length (Le). Not considering the localized head losses overestimated the maximum lateral lengths of $25.7 \%$, for the pressure compensating emitters, and of $9.5 \%$, for the not pressure compensating emitters. The calculation of localized head loss using the potential model to estimate the $\mathrm{K}$ value in function of obstruction index, showed good results, comparable to those obtained with the fitted $\mathrm{K}$ value by the data observed at the laboratory, resulting in small variations in the estimate of maximum lateral lengths for those two procedures.
\end{abstract}

KEYWORDS: drip irrigation, kinetic load coefficient, localized head loss, obstruction index, equivalent length.

\footnotetext{
${ }^{1}$ Eng ${ }^{0}$ Agrícola, M.Sc. em Irrigação e Drenagem, Departamento de Engenharia de Biossistemas, ESALQ/USP, Piracicaba - SP, Fone: (0XX19) 3447.8576, anthonnyleto@ hotmail.com.

${ }^{2}$ Eng ${ }^{0}$ Agrônomo, Prof. Titular, Departamento de Engenharia de Biossistemas, ESALQ/USP, Piracicaba - SP, frizzone@esalq.usp.br.

${ }^{3}$ Engo Agrícola, M.Sc. em Irrigação e Drenagem, Departamento de Engenharia de Biossistemas, ESALQ/USP, Piracicaba - SP, orettore@esalq.usp.br.

${ }^{4}$ Eng $^{\circ}$ Agrônomo, Prof. Associado, Departamento de Ciências Exatas, ESALQ/USP, Piracicaba - SP, jhmirand@esalq.usp.br.

Recebido pelo Conselho Editorial em: 20-2-2009

Aprovado pelo Conselho Editorial em: 13-1-2010
} 


\section{INTRODUÇÃO}

Para que os sistemas de irrigação por gotejamento atinjam alta eficiência, devem ser observados os fatores que afetam a uniformidade de emissão, como a variação de vazão dos emissores ao longo da lateral, decorrente da variação de pressão por perda de carga e por desnível geométrico, a variação de vazão por variabilidade de fabricação dos emissores, a obstrução de emissores e as variações de temperatura da água ao longo da lateral (PROVENZANO \& PUMO, 2004).

A perda de carga localizada ocorre quando o fluido sofre alguma perturbação brusca em seu escoamento, por algum elemento inserido no tubo. No dimensionamento do sistema de irrigação por gotejamento, é imprescindível determinar as perdas totais de cargas que ocorrem ao longo da tubulação (perda de carga linear mais perdas localizadas). As perdas localizadas, provocadas pelos emissores dentro da tubulação, muitas vezes, são desprezadas (AL-AMOUD, 1995). Entretanto, elas são significativas e de grande importância para se dimensionar o comprimento máximo das linhas laterais (KELLER \& KARMELI, 1974; BAGARELLO et al., 1997; RETTORE NETO et al., 2009a), sobretudo quando se deseja otimizar o custo total do projeto.

A perda de carga localizada, causada pelo emissor, depende da área que ele ocupa na tubulação e de sua forma geométrica, existindo, comumente, três tipos de conexões: sobre a linha (on-line), na linha (in-line) e emissores integrados dentro da linha (BAGARELLO et al., 1997; ALVES \& PORTO, 2002; CARDOSO \& FRIZZONE, 2007; RETTORE NETO et al., 2009b) propuseram equações, através de modelagem matemática, para calcular a perda de carga localizada provocada por emissores inseridos e integrados no tubo. Os modelos basearam-se no teorema de Bélanger, utilizando relações geométricas do tubo e dos emissores.

Os fabricantes disponibilizam, aos projetistas de sistemas de irrigação por gotejamento, vários modelos de emissores, porém, em geral, não apresentam, nos catálogos de características do produto, informações sobre as relações dimensionais dos emissores e a perda de carga localizada que eles provocam no escoamento através da tubulação. Essas relações dimensionais são importantes para se prever a perda de carga localizada durante a execução do projeto hidráulico.

Este trabalho foi desenvolvido com o objetivo de relacionar a perda de carga localizada em gotejadores coaxiais integrados em tubos de polietileno com a geometria da seção de escoamento no ponto de inserção, mediante a utilização de um índice de obstrução calculado pelas relações entre as áreas de escoamento no tubo uniforme e na passagem pelo gotejador.

\section{MATERIAL E MÉTODOS}

O trabalho foi realizado no laboratório de Irrigação do Departamento de Engenharia de Biossistemas da Escola Superior de Agricultura "Luiz de Queiroz" (ESALQ), da Universidade de São Paulo (USP), Piracicaba - SP. Os tubos utilizados no experimento foram de polietileno, de pequenos diâmetros, com emissores coaxiais, do tipo "bobi", fabricados pela Amanco e pela NaanDan. As suas características operacionais são apresentadas na Tabela 1.

TABELA 1. Características dos tubos e emissores utilizados nos ensaios. Tube and emitter characteristics used in the experiment.

\begin{tabular}{ccccccc}
\hline $\begin{array}{c}\text { Diâmetro } \\
\text { Nominal }(\mathrm{mm})\end{array}$ & $\begin{array}{c}\text { Diâmetro Interno }(\mathrm{mm}) \\
\text { Into }\end{array}$ & Fabricante & Modelo & $\begin{array}{c}\text { Pressão de } \\
\text { Operação }(\mathrm{kPa})\end{array}$ & $\begin{array}{c}\text { Vazão } \\
\left(\mathrm{L} \mathrm{h}^{-1}\right)\end{array}$ & $\begin{array}{c}\text { Espaçamento entre } \\
\text { Emissores }(\mathrm{m})\end{array}$ \\
\hline 16 & 13,70 & Amanco & Drip AC & $100-450$ & $2,3-4,0$ & 0,90 \\
16 & 13,60 & Amanco & Drip & $100-750$ & $1,4-4,5$ & 0,95 \\
16 & 13,00 & NaanDan & Naan PC & $100-350$ & $1,3-3,8$ & 1,00 \\
16 & 13,30 & NaanDan & Naan Tif & $100-350$ & $1,0-4,0$ & 1,00 \\
\hline
\end{tabular}

PC e AC - autocompensante. 
Para os ensaios, utilizou-se uma bancada composta por um reservatório de água de $1.000 \mathrm{~L}$, uma motobomba modelo C3000-KSB, ligada a um inversor de frequência Siemens, modelo Micromaster 420, com a finalidade de manter a rotação do motor constante; três registros de gaveta, com o propósito de regular a pressão no inicio do tubo gotejador e um medidor de vazão magnético indutivo. Para o monitoramento da pressão de operação do sistema, utilizou-se um manômetro digital com faixa de trabalho de 0 a $1.500 \mathrm{kPa}$, com precisão de $1 \mathrm{kPa}$. A determinação da perda de carga distribuída foi realizada por meio de manômetro diferencial em "U", contendo líquido manométrico de densidade $13,6 \mathrm{~g} \mathrm{~cm}^{-3}$ e divisão de escala de $1,0 \mathrm{~mm}$. O esquema de montagem do experimento é mostrado na Figura 1.

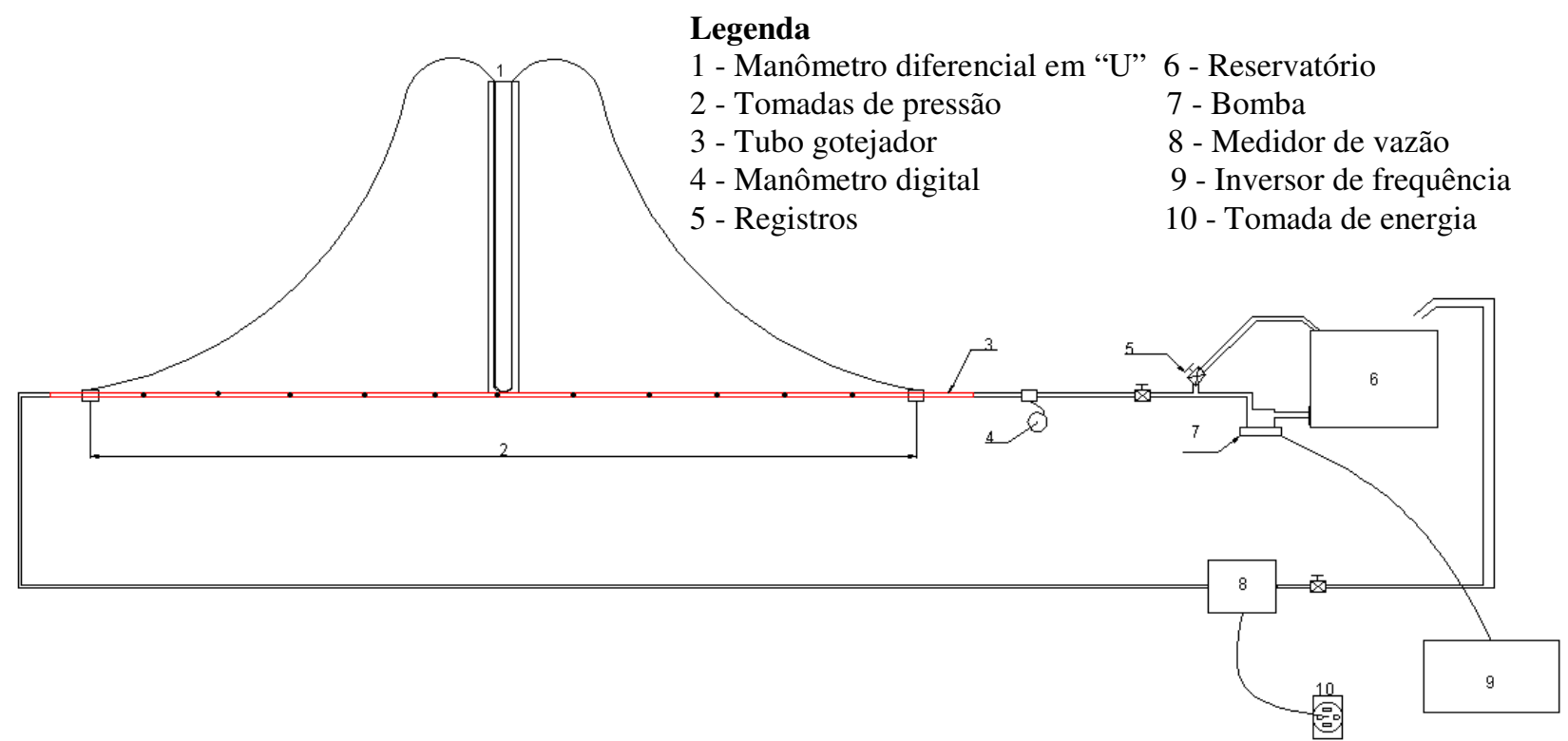

FIGURA 1. Croqui da instalação experimental para a determinação da perda de carga. Layout of the experimental installation for determination of head loss.

O diâmetro interno do tubo gotejador foi determinado em 30 anéis de $1 \mathrm{~cm}$ de comprimento, utilizando-se de um projetor óptico de perfil Starrett, modelo HB 400. Na Tabela 2, são apresentados os diâmetros e as espessuras das paredes dos tubos utilizados nos ensaios.

TABELA 2. Características dos tubos gotejadores ensaiados. Drip pipes characteristics used in the experiment.

\begin{tabular}{cccccc}
\hline \multirow{2}{*}{ Modelos } & \multicolumn{2}{c}{ Diâmetro Interno $(\mathrm{mm})$} & & \multicolumn{2}{c}{ Espessura da Parede $(\mathrm{mm})$} \\
\cline { 2 - 3 } \cline { 5 - 6 } & Média & Desvio-Padrão & & Média & Desvio-Padrão \\
\hline Amanco Drip AC & 13,70 & 0,18 & & 1,04 & 0,052 \\
Amanco Drip & 13,60 & 0,13 & & 1,07 & 0,072 \\
Naan PC & 13,00 & 0,18 & & 0,98 & 0,043 \\
Naan TIF & 13,30 & 0,13 & & 0,99 & 0,040 \\
\hline
\end{tabular}

Para obstruir a passagem da água na saída do orifício dos gotejadores, fez-se vedação dos emissores com massa "epox", envolvendo-os posteriormente com uma fita adesiva e borracha flexível para garantir a vedação.

Os orifícios para tomada de pressão, no início e no final da tubulação, foram confeccionados a uma distância dos emissores igual à metade do espaçamento entre eles. Inicialmente, foram feitos furos primários para a demarcação do local e, em seguida, o furador foi aquecido para fazer a perfuração definitiva. Para minimizar a ocorrência de rebarbas e retirar os resíduos de polietileno, girava-se o furador suavemente para os lados, movimentando-o verticalmente. 
Para preparar as tomadas de pressão, foram utilizadas luvas de PVC, anéis de silicone e presilhas parafusadas lentamente, tendo-se o cuidado para não ocasionar deformação do tubo e causar distúrbios no escoamento da água e perdas de carga adicionais.

Utilizaram-se segmentos de tubos contendo 11 gotejadores, com comprimentos de $9,90 \mathrm{~m} \mathrm{e}$ 10,45 m para os tubos da Amanco e $11 \mathrm{~m}$ de comprimento para os tubos da NaanDan.

O ensaio consistia na determinação da vazão que passava pelo tubo e a perda de carga ocorrida entre os dois pontos, observada no manômetro diferencial. A vazão iniciou-se com $8 \times 10^{-2} \mathrm{~m}^{3} \mathrm{~h}^{-1}$, continuando com intervalos de valores crescentes, de $8 \times 10^{-2} \mathrm{~m}^{3} \mathrm{~h}^{-1}$, até chegar $1,6 \mathrm{~m}^{3} \mathrm{~h}^{-1}$, obtendo-se um número de 20 distintas vazões para cada tubo avaliado. No decorrer dos ensaios, foram monitoradas as temperaturas, por meio de um termômetro de mercúrio, com a finalidade de se fazer a correção da viscosidade cinemática da água no cálculo da perda de carga localizada.

Para a tubulação em nível, contendo 11 emissores vedados, considerou-se a perda de carga no segmento de tubo, como sendo a diferença de leitura de pressão entre os dois pontos avaliados. A perda de carga observada no tubo com emissor foi quantificada em função da vazão, utilizando-se de um modelo tipo potência, na forma da eq.(1):

$$
\mathrm{Hf}_{\mathrm{te}}=\mathrm{aQ}^{\mathrm{b}}
$$

sendo,

$\mathrm{Hf}_{\mathrm{te}}$ - perda de carga no tubo com emissor, $\mathrm{m}$;

$\mathrm{Q}-$ vazão, $\mathrm{m}^{3} \mathrm{~s}^{-1}, \mathrm{e}$

$\mathrm{a}$ e b - constantes.

A perda de carga provocada pelo emissor foi determinada em função da diferença entre a perda de carga do tubo com o emissor e a perda de carga do tubo calculada pela equação de Darcy-Weisbach, com $\mathrm{f}$ determinado para tubo sem emissor, e do número de emissores no segmento de comprimento [eq.(2)]:

$$
\mathrm{Hf}_{\mathrm{e}}=\frac{\mathrm{Hf}_{\mathrm{te}}-\mathrm{Hf}_{\mathrm{t}}}{\mathrm{N}}
$$

sendo,

$\mathrm{Hf}_{\mathrm{e}}$ - perda de carga no emissor, $\mathrm{m}$;

$\mathrm{Hf}_{\mathrm{t}}$ - perda de carga no tubo, $\mathrm{m}$, e

$\mathrm{N}$ - número de emissores.

A perda de carga no emissor foi ajustada em função da vazão, utilizando-se de um modelo potencial [eq.(3)]:

$$
\mathrm{Hf}_{\mathrm{e}}=\alpha \mathrm{Q}^{\mathrm{K}}
$$

em que,

$\alpha$ - constante;

$\mathrm{Q}$ - vazão, $\mathrm{m}^{3} \mathrm{~s}^{-1}, \mathrm{e}$

$\mathrm{K}$ - constante.

Para expressar a perda de carga localizada no emissor, em termos de comprimento equivalente de tubulação (Le), utilizou-se das equações de Darcy-Weisbach eq.(4), para a perda de carga localizada, a eq.(5), conforme REDDY (2003). Rearranjando os termos dessas equações, Le (m) pode ser expresso pela eq.(6):

$$
\mathrm{Hf}=f \frac{\mathrm{L}_{\mathrm{t}}}{\mathrm{D}} \frac{\mathrm{V}^{2}}{2 \mathrm{~g}}
$$




$$
\begin{aligned}
& \mathrm{Hf}_{\mathrm{L}}=\mathrm{K} \frac{\mathrm{V}^{2}}{2 \mathrm{~g}} \\
& \mathrm{Le}=\frac{\mathrm{K} \mathrm{D}}{\mathrm{f}}
\end{aligned}
$$

sendo,

Hf - perda de carga, m;

$\mathrm{L}_{\mathrm{t}}$ - comprimento do tubo, $\mathrm{m}$;

D - diâmetro interno do tubo, m;

$\mathrm{V}$ - velocidade média de escoamento, $\mathrm{m} \mathrm{s}^{-1}$;

$\mathrm{g}$ - aceleração da gravidade, $\mathrm{m} \mathrm{s}^{-2}$;

$f$ - fator de atrito;

$\mathrm{Hf}_{\mathrm{L}}$ - perda de carga localizada, $\mathrm{m}$;

$\mathrm{K}$ - coeficiente de carga cinética, e

Le - comprimento equivalente, $\mathrm{m}$.

PROVENZANO \& PUMO (2004) e PROVENZANO et al. (2005) desenvolveram uma relação potencial que permite prever o valor de $\mathrm{K}$, em função da razão entre o diâmetro da seção transversal do tubo $\left(\mathrm{D}_{\mathrm{t}}\right)$ e o diâmetro equivalente da seção de escoamento dentro do emissor $\left(\mathrm{D}_{\mathrm{g}}\right)$, para o domínio $1,04<\mathrm{D}_{\mathrm{t}} / \mathrm{D}_{\mathrm{g}}<1,16\left(1,08<\mathrm{A}_{\mathrm{t}} / \mathrm{A}_{\mathrm{g}}<1,35\right)$. Para ampliar o domínio desta função para $1,08<\mathrm{A}_{\mathrm{t}} / \mathrm{A}_{\mathrm{g}}<1,89$, agruparam-se os resultados deste experimento com os resultados de PROVENZANO et al. (2005) e ajustou-se uma nova função potência aos pares de pontos (K, IO), sendo IO um índice de obstrução definido por $\mathrm{IO}=(1-\mathrm{r})^{2} / \mathrm{r}^{2}$ e $\mathrm{r}$ é a razão de obstrução definida por $\mathrm{r}=\mathrm{A}_{\mathrm{g}} / \mathrm{A}_{\mathrm{t}}$, resultando na eq.(7):

$$
\mathrm{K}=1,68\left(\frac{1}{\mathrm{r}}-1\right)^{1,29}
$$

\section{RESULTADOS E DISCUSSÃO}

$\mathrm{Na}$ Figura 2, apresenta-se a perda de carga nos tubos gotejadores $\left(\mathrm{Hf}_{\mathrm{te}}\right)$, contendo 11 emissores, vedados, em função da vazão. Observa-se que há relação potencial entre a vazão e a perda de carga, com $\mathrm{R}^{2}$ acima de 0,9996 , significando que $99,96 \%$ das variações da perda de carga são explicadas pela variação de vazão. Os expoentes da vazão variaram entre 1,7657 e 1,8484, indicando o escoamento turbulento em tubos lisos.

Utilizando as equações obtidas pelas linhas de tendência e fixando uma vazão de $20 \times 10^{-5} \mathrm{~m}^{3} \mathrm{~s}^{-1}$, obtêm-se as seguintes perdas de carga unitárias no tubo gotejador: Amanco Drip $\mathrm{AC}=0,293 \mathrm{~m} \mathrm{~m}^{-1}$; Amanco Drip $=0,213 \mathrm{~m} \mathrm{~m}^{-1}$, Naan PC $=0,344 \mathrm{~m} \mathrm{~m}^{-1}$ e Naan Tif, 0,202 $\mathrm{m} \mathrm{m}^{-1}$. Observa-se que os tubos gotejadores autocompensados (Amanco Drip AC e Naan PC) proporcionam maiores perdas unitárias de carga que os tubos gotejadores Amanco Drip e Naan Tif. Considerando-se que os diâmetros dos tubos e o espaçamento entre os emissores não apresentam diferenças apreciáveis, o tubo gotejador Amanco Drip AC (autocompensado) produziu uma perda de carga 37,6\% maior que o Amanco Drip (não autocompensado), e o Naan PC (autocompensado) produziu uma perda de carga 70,3\% maior que o Naan Tif (não autocompensado). Esses acréscimos na perda de carga estão relacionados à geometria do gotejador, uma vez que o dispositivo de regulação de pressão ocupa certa fração da área de escoamento. 

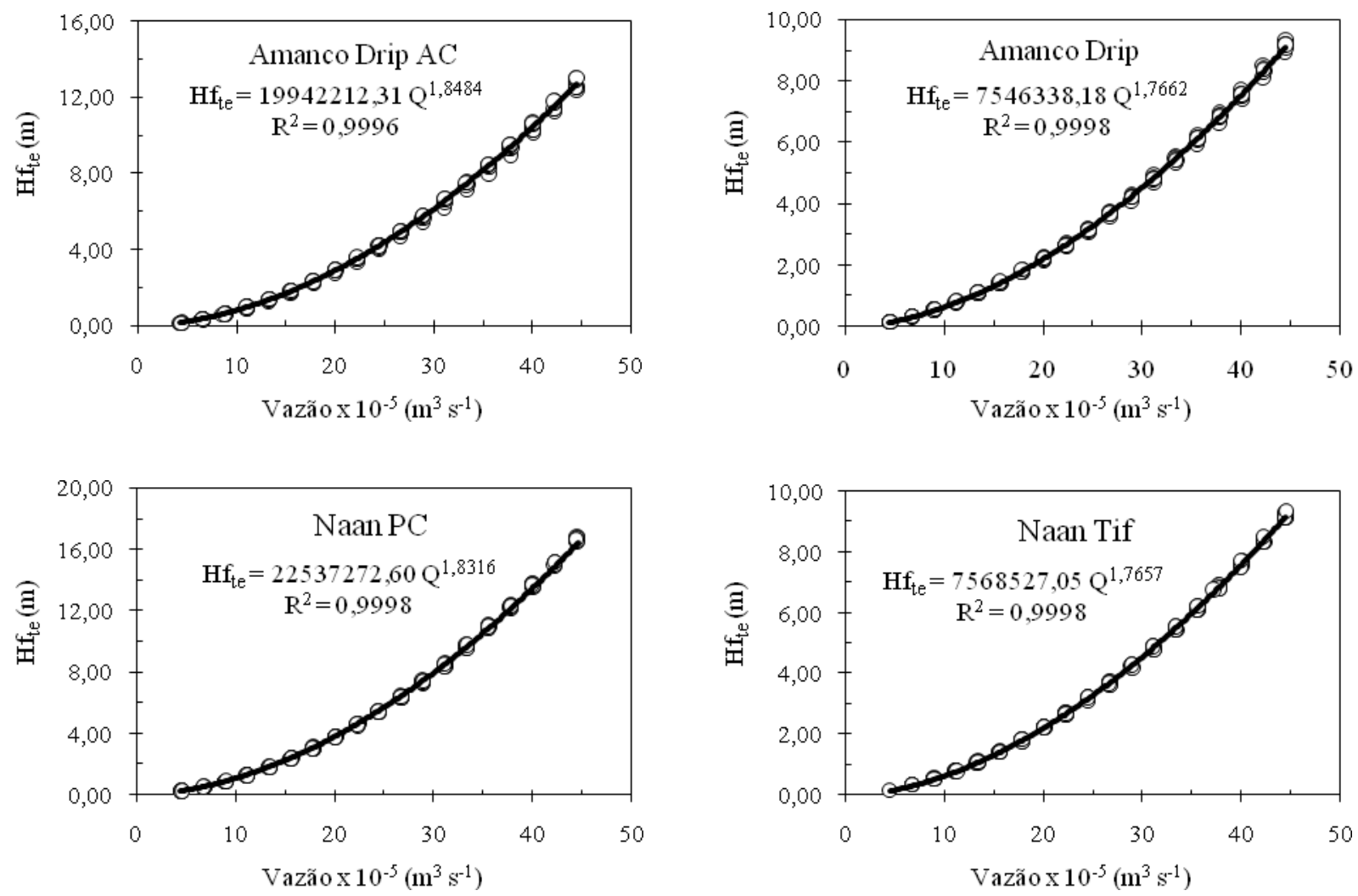

FIGURA 2. Perda de carga nos tubos gotejadores contendo 11 emissores, vedados, em função da vazão, para os seguintes comprimentos de tubo: Amanco Drip $\mathrm{AC}=9,9 \mathrm{~m}$; Amanco Drip $=10,4 \mathrm{~m}$; Naan $\mathrm{PC}=11 \mathrm{~m}$; Naan Tif $=11 \mathrm{~m}$. Head loss in the pipe with 11 emitters, closed, for the following lengths of pipe: Amanco Drip AC = 9,9 m; Amanco Drip = 10,4 m; Naan PC = $11 \mathrm{~m}$; Naan Tif $=11 \mathrm{~m}$.

Na Figura 3, estão apresentadas as linhas de tendência da perda de carga localizada nos emissores $\left(\mathrm{Hf}_{\mathrm{e}}, \mathrm{m}\right)$, em função da vazão $\left(\mathrm{Q}, \mathrm{m}^{3} \mathrm{~s}^{-1}\right)$, para os dados experimentais, utilizando os emissores Amanco Drip AC, Amanco Drip, Naan PC e Naan Tif, com as respectivas equações e os coeficientes de determinação $\left(R^{2}\right)$. Para todas as curvas, os valores de $R^{2}$ foram superiores a $96 \%$, mostrando que, no mínimo, $96 \%$ das variações da perda de carga observada podem ser explicadas pelas variações da vazão. A maior dispersão dos valores de perda de carga ocorreu nos ensaios com o gotejador Naan Tif, principalmente para vazões superiores a $20 \times 10^{-5} \mathrm{~m}^{3} \mathrm{~s}^{-1}$.

Observa-se que ocorreu maior perda de carga nos modelos de emissores autocompensantes (Amanco Drip AC e Naan PC). Tomando-se como referência a vazão de $20 \times 10^{-5} \mathrm{~m}^{3} \mathrm{~s}^{-1}$, as perdas de cargas estimadas nos emissores foram: Amanco Drip AC: 0,114 m; Naan PC: 0,134 m; Naan Tif: 0,015 m; Amanco Drip: 0,038 m.

Entre os gotejadores autocompensantes, o modelo Naan PC apresentou maior perda de carga localizada (uma diferença de 17,4\% em relação ao Amanco Drip PC), por apresentar menor diâmetro equivalente na seção de escoamento através do emissor. Entre os gotejadores não autocompensantes, o modelo Amanco Drip apresentou maior perda de carga localizada (uma diferença de 153,3\% em relação ao Naan Tif), decorrente do menor diâmetro da seção de escoamento através do gotejador.

Para a vazão de $20 \times 10^{-5} \mathrm{~m}^{3} \mathrm{~s}^{-1}$, analisando-se os gotejadores Amanco, observou-se que um aumento de 1,79 vez no grau de obstrução resultou no aumento de três vezes na perda de carga localizada (Tabela 3). Nos gotejadores Naan, um aumento de 2,2 vezes no grau de obstrução proporcionou um aumento de 8,9 vezes na perda de carga localizada. 

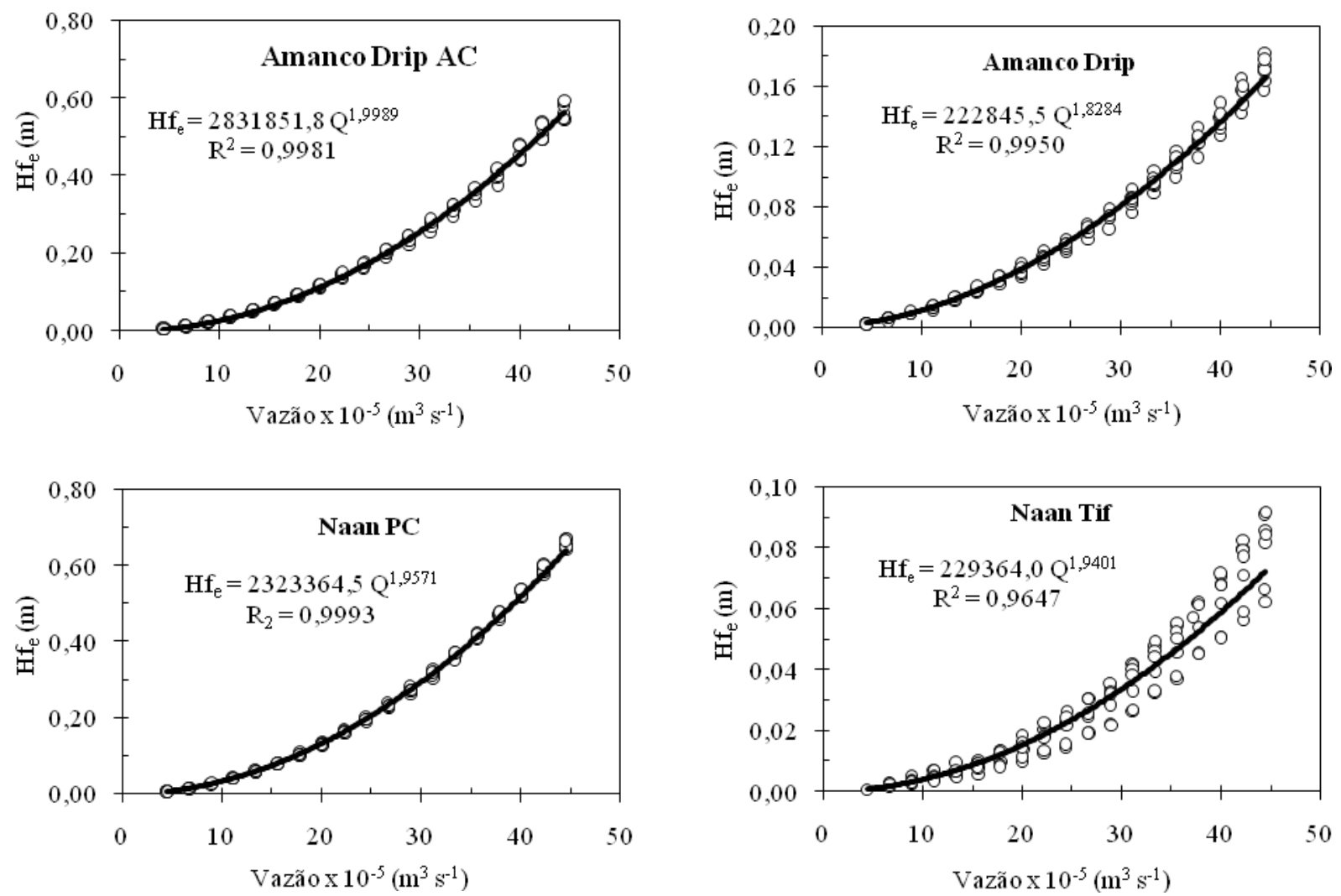

FIGURA 3. Perda de carga localizada no emissor, em função da vazão. Local head loss at the emitter in relation to flow.

TABELA 3. Característica dos emissores. Emitters characteristics.

\begin{tabular}{lcccccc}
\hline Emissores & $\mathrm{At}\left(\mathrm{mm}^{2}\right)$ & $\mathrm{Dg}(\mathrm{mm})$ & $\mathrm{Ag}\left(\mathrm{mm}^{2}\right)$ & $\mathrm{Dg} / \mathrm{Dt}$ & $\mathrm{Ag} / \mathrm{At}$ & $\mathrm{GO}$ \\
\hline Amanco Drip AC & 147,63 & $9,43^{*}$ & 77,90 & 0,688 & 0,528 & 0,472 \\
Amanco Drip & 144,84 & 11,65 & 106,60 & 0,858 & 0,736 & 0,264 \\
Naan PC & 136,35 & $9,01^{*}$ & 76,97 & 0,692 & 0,577 & 0,423 \\
Naan Tif & 139,56 & 11,98 & 112,72 & 0,899 & 0,808 & 0,192
\end{tabular}

* Dg - diâmetro interno equivalente da seção de escoamento através do gotejador, calculado a partir do raio hidráulico $(\mathrm{Dg}=4 \mathrm{Rh})$

* GO - grau de obstrução (= 1- Ag/At).

A perda de carga localizada aumentou com a redução da área de escoamento no local de inserção do gotejador, conforme também foi observado nos trabalhos experimentais realizados por AL-AMOUD (1995), BAGARELLO et al. (1997), PROVENZANO \& PUMO (2004), PROVENZANO et al. (2005); CARDOSO \& FRIZZONE (2007). Esse fato sugere que a perda de carga localizada nos gotejadores pode ser analisada em função da morfologia (forma e tamanho) do emissor.

Os gotejadores autocompensantes produzem maior perda de carga do que os gotejadores convencionais; entretanto, são preferidos pelos projetistas de irrigação localizada, especialmente em terrenos com topografia acidentada, por facilitarem os cálculos hidráulicos e garantirem melhor uniformidade na distribuição de água, embora possam aumentar a perda de carga nas linhas laterais, o custo de aquisição e o custo do bombeamento.

$\mathrm{Na}$ Figura 4, mostra-se a perda de carga nos emissores em função da carga cinética $\left(\mathrm{V}^{2} / 2 \mathrm{~g}, \mathrm{~m}\right)$; verifica-se que, nos modelos não autocompensantes, ocorreram maiores dispersões nas perdas de carga observadas, aumentando com o aumento da velocidade. Observa-se, também, que a tendência das curvas é semelhante para os quatro emissores estudados. 

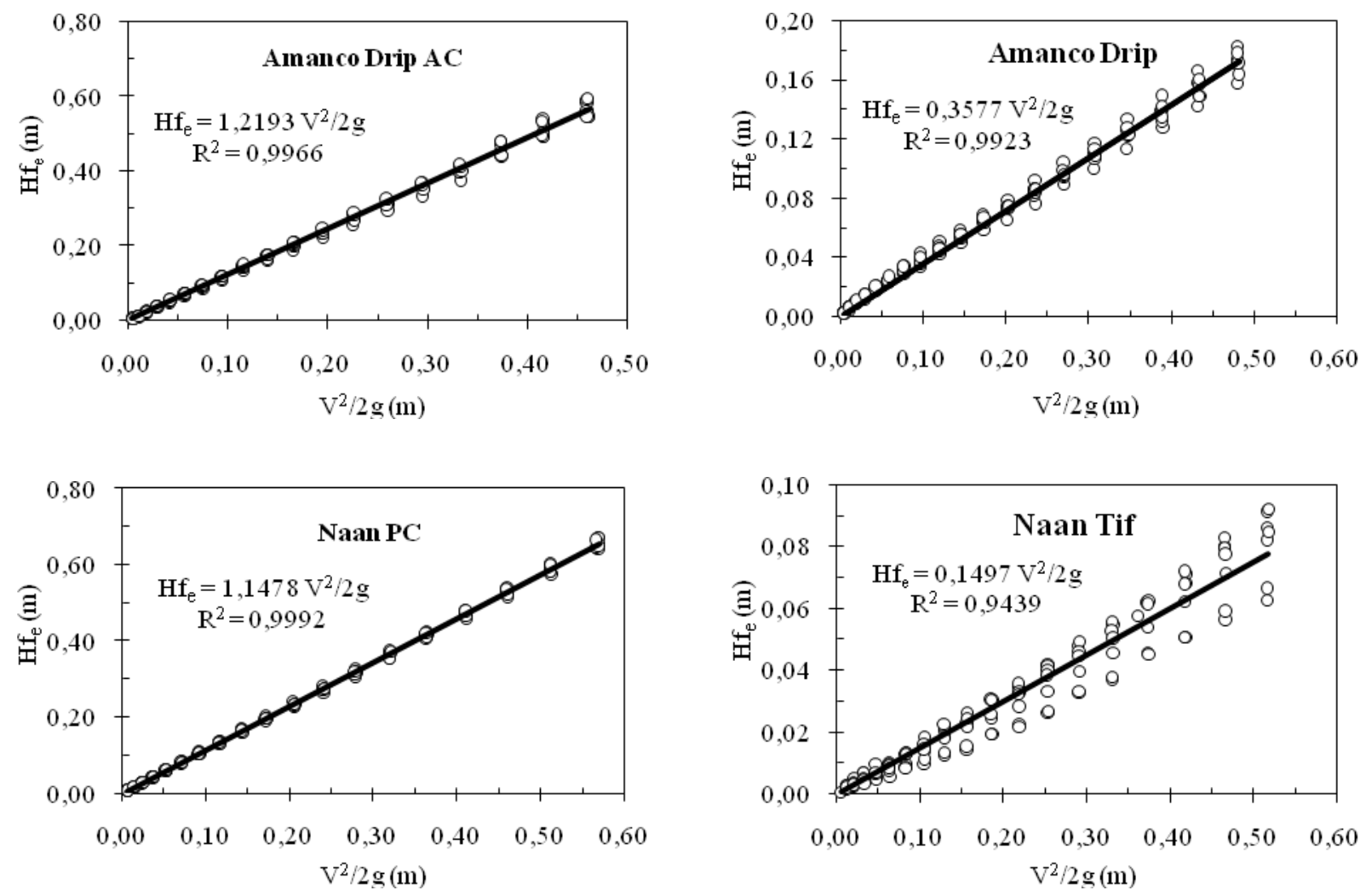

FIGURA 4. Perda de carga em função da carga cinética. Head loss in relation to kinetic load.

Observa-se que o emissor Naan Tif apresentou o menor coeficiente de carga cinética ( $\mathrm{K}=0,1497)$, e o Amanco Drip AC, o maior coeficiente $(\mathrm{K}=1,2193)$. Para os emissores Naan Tif, obteve-se maior dispersão dos valores de perda de carga nas amostras analisadas, o que pode ser causado por alguma variabilidade na geometria do emissor. A Tabela 4 mostra a amplitude de variação dos valores de $(\mathrm{K})$ e o intervalo de confiança desses valores a $95 \%$ de probabilidade, indicando que existem $95 \%$ de probabilidade de esse intervalo conter a verdadeira média. Observa-se que os gotejadores autocompensados proporcionaram menor variabilidade no coeficiente $\mathrm{K}$.

TABELA 4. Valores máximo e mínimo de $\mathrm{K}$ para os emissores ensaiados. Maximum and minimum values of $K$ for emitter tested.

\begin{tabular}{lccc}
\hline \multirow{2}{*}{ Emissor } & \multicolumn{3}{c}{ Valores de K da Regressão } \\
\cline { 2 - 4 } & Médio & Amplitude & IC (95\%)* \\
\hline Amanco Drip AC & 1,2193 & $1,1880-1,2744$ & $1,2115-1,2271$ \\
Amanco Drip & 0,3577 & $0,3284-0,3848$ & $0,3545-0,3612$ \\
Naan PC & 1,1478 & $1,1399-1,1749$ & $1,1445-1,1515$ \\
Naan Tif & 0,1497 & $0,1165-0,1723$ & $0,1457-0,1538$ \\
\hline
\end{tabular}

* Intervalo de confiança a $95 \%$.

O coeficiente de carga cinética $(\mathrm{K})$ depende do número de Reynolds $(\mathrm{R})$ e das características geométricas do elemento obstrutor. No escoamento turbulento, o coeficiente K está mais fortemente relacionado à geometria da seção de escoamento (AZEVEDO NETO et al., 1998). Na Figura 5, observa-se que os valores de K, para cada emissor estudado, variaram pouco, com R > 10000 . 


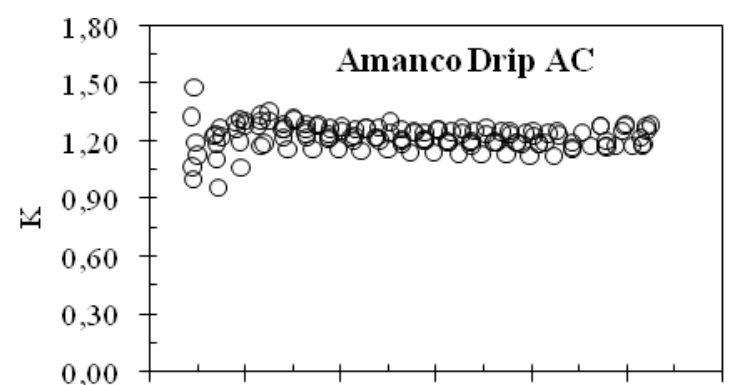

$0 \quad 100002000030000400005000060000$

$\mathrm{R}$

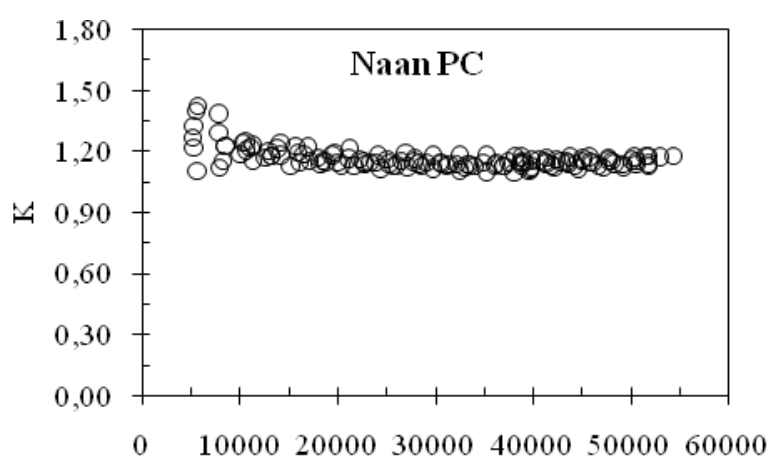

$\mathrm{R}$
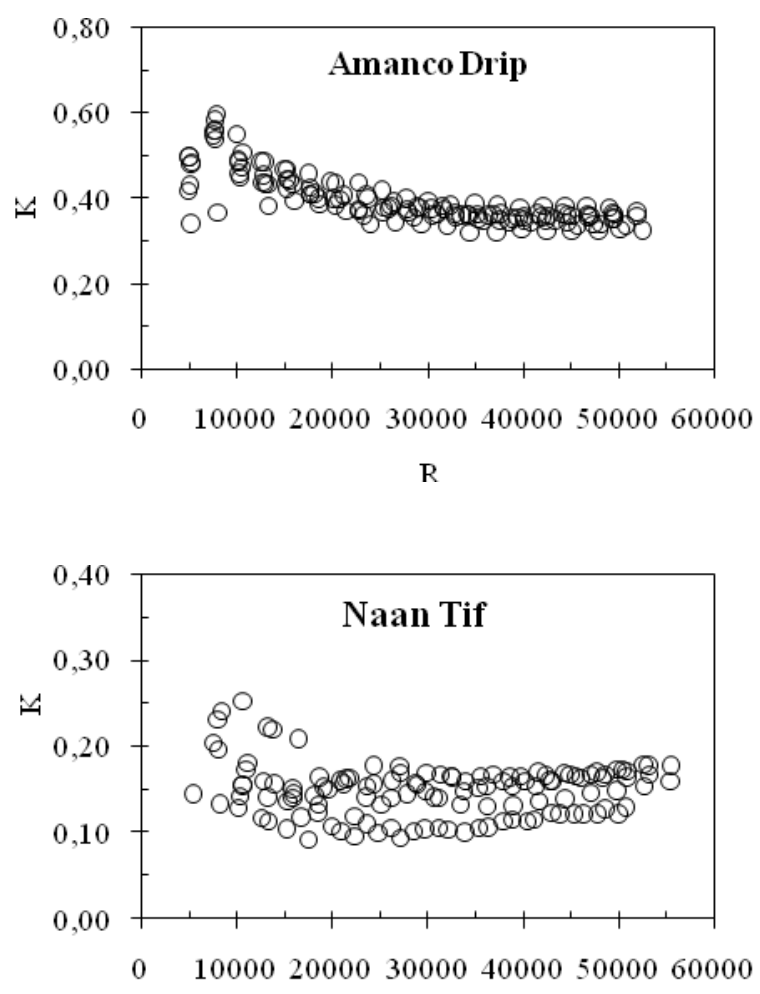

$\mathrm{R}$

FIGURA 5. Valores observados de (K) em função de (R). Observed values of $\mathbf{K}$ in relation to $\mathbf{R}$.

Como resultado, pode-se considerar que o efeito das forças viscosas é desprezível para $\mathrm{R}>10.000$, e $\mathrm{K}$ depende, em grande parte, da forma e do tamanho do elemento obstrutor, e que um valor médio de $\mathrm{K}$ pode ser previsto por um índice de obstrução (Figura 6). As aproximações para a perda de carga localizada, em termos de comprimento equivalente (Le), possuem validades práticas (JUANA et al., 2002 a ,b), porém deve ser observado que $f$ varia ao longo da linha lateral com a variação da vazão. Pode também variar em uma lateral específica, em decorrência da variação da pressão de entrada que afeta a vazão de emissores não autocompensados. Consequentemente, Le não é constante para um dado valor de K e nem K é constante para um dado valor de Le.

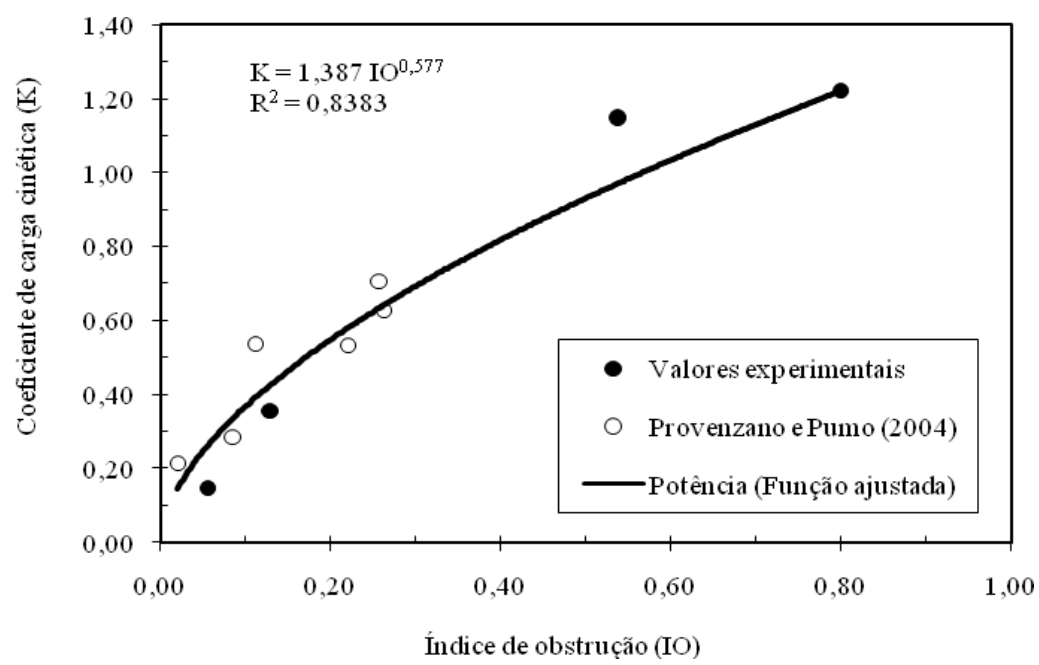

FIGURA 6. Relação entre o índice de obstrução e o coeficiente de carga cinética. Relation between obstruction index and kinetic load coefficient. 
Entretanto, como critério prático, pode-se assumir um comprimento equivalente constante para cada gotejador igualmente espaçado, expressando-se Le $(\mathrm{m})$ em função de D $(\mathrm{m})$ e Q $\left(\mathrm{m}^{3} \mathrm{~s}^{-1}\right)$ e $\mathrm{K}$, calculado pela relação expressa na Figura 6, o que resulta na eq.(8):

$$
\mathrm{Le}=2,2139 \mathrm{~K} \mathrm{D}^{4,75} \mathrm{Q}^{-1,75} \eta^{-0,25}
$$

A perda total de carga na linha de gotejadores pode ser calculada em função da vazão de entrada $\left(\mathrm{Q}\right.$, em $\left.\mathrm{m}^{3} \mathrm{~s}^{-1}\right)$, do diâmetro interno da tubulação $(\mathrm{D}$, em $\mathrm{m})$, do comprimento da tubulação $\left(\mathrm{L}_{\mathrm{t}}\right.$, em $\mathrm{m}$ ), do espaçamento entre os gotejadores (Se, em $\mathrm{m}$ ), do comprimento equivalente (Le, em $\mathrm{m})$, da viscosidade cinemática da água $\left(\eta, \mathrm{em} \mathrm{m}^{2} \mathrm{~s}^{-1}\right)$ e do fator de redução da perda de carga para tubulações com múltiplas saídas (F).

$\mathrm{Na}$ Tabela 5, apresentam-se a vazão nominal de cada gotejador (qn), o seu espaçamento na linha lateral $(\mathrm{Se})$ e a pressão de serviço indicada pelo fabricante (Hs). Para os gotejadores não autocompensados, considerou-se, como critério de dimensionamento, a variação máxima de vazão de 10\%. Para os gotejadores autocompensados, considerou-se a variação máxima de pressão na lateral igual a $200 \mathrm{kPa}$. Pode-se observar que houve variação da perda de carga localizada nos diferentes emissores e que a consideração dessa perda de carga é importante, especialmente para os gotejadores autocompensados. A desconsideração da perda de carga localizada levou a uma superestimativa do comprimento máximo da linha lateral de até $25,7 \%$, para os gotejadores autocompensados; já para os gotejadores não autocompensados, a superestimativa foi de, no máximo, $9,5 \%$.

Na Figura 7, apresenta-se uma comparação entre os comprimentos equivalentes obtidos com os dados experimentais e o comprimento equivalente calculado pela eq.(8); nela pode-se observar que, para os emissores Amanco Drip AC e Amanco Drip, os valores de Le, calculados por essa equação, ajustaram-se melhor aos valores observados. Já nos emissores Naan PC e Naan Tif, o modelo apresentou desvios significativos. Para o emissor Naan PC, a Eq. (8) subestimou o comprimento equivalente em 25,5\% e, para o emissor Naan Tif, superestimou em 60,3\%. Para demonstrar a importância da perda de carga localizada em projetos de irrigação, aplicou-se essa consideração para dimensionar o comprimento máximo de uma linha lateral, utilizando-se de três procedimentos (Tabela 5) para os tubos gotejadores estudados.

TABELA 5. Cálculo do comprimento máximo de uma lateral. Calculation of maximum lateral length.

\begin{tabular}{|c|c|c|c|c|c|c|c|c|c|c|c|c|}
\hline \multirow{3}{*}{ Parâmetros } & \multicolumn{12}{|c|}{ Emissor } \\
\hline & \multicolumn{3}{|c|}{ Naan PC } & \multicolumn{3}{|c|}{ Amanco Drip AC } & \multicolumn{3}{|c|}{ Naan Tif +} & \multicolumn{3}{|c|}{ Amanco Drip ++ } \\
\hline & I & II & III & I & II & III & $\mathrm{I}$ & II & III & I & II & III \\
\hline $\mathrm{qn}\left(\mathrm{L} \mathrm{h}^{-1}\right)$ & 3,8 & 3,8 & 3,8 & 4,0 & 4,0 & 4,0 & 3,6 & 3,6 & 3,6 & 4,2 & 4,2 & 4,2 \\
\hline $\operatorname{Se}(\mathrm{m})$ & 0,5 & 0,5 & 0,5 & 0,5 & 0,5 & 0,5 & 0,5 & 0,5 & 0,5 & 0,5 & 0,5 & 0,5 \\
\hline Hs $(\mathrm{kPa})$ & $100-350$ & $100-350$ & $100-350$ & $100-300$ & $100-300$ & $100-300$ & 100 & 100 & 100 & 100 & 100 & 100 \\
\hline Hinic $(\mathrm{kPa})$ & 300 & 300 & 300 & 300 & 300 & 300 & 110 & 110 & 110 & 110 & 110 & 110 \\
\hline Hfim (kPa) & 101 & 102 & 102 & 101 & 101 & 101 & 92 & 92 & 92 & 87 & 87 & 87 \\
\hline $\mathrm{Qe}\left(\mathrm{L} \mathrm{h}^{-1}\right)$ & $1.018,4$ & 775,2 & 798,0 & $1.136,0$ & 844,0 & 844,0 & 431,1 & 417,2 & 402,8 & 515,5 & 466,6 & 458,4 \\
\hline $\mathrm{Hf}(\mathrm{m})$ & 19,9 & 9,5 & 10,3 & 19,9 & 9,0 & 9,0 & 1,8 & 1,6 & 1,5 & 2,2 & 1,8 & 1,7 \\
\hline $\mathrm{Hf}_{\mathrm{e}}(\mathrm{m})$ & --- & 10,3 & 9,5 & --- & 10,9 & 10,9 & --- & 0,2 & 0,3 & --- & 0,5 & 0,6 \\
\hline $\mathrm{Hf}_{\mathrm{te}}(\mathrm{m})$ & 19,9 & 19,8 & 19,8 & 19,9 & 19,9 & 19,9 & 1,8 & 1,8 & 1,8 & 2,2 & 2,3 & 2,3 \\
\hline $\mathrm{N}$ & 268 & 204 & 210 & 284 & 211 & 211 & 123 & 118 & 114 & 127 & 115 & 112 \\
\hline Lmáx (m) & 134 & 102 & 105 & 142 & 105,5 & 105,5 & 61,5 & 59 & 57 & 63,5 & 57,5 & 56,5 \\
\hline Lvar (\%) & --- & $-23,9$ & $+2,9$ & --- & $-25,7$ & 0 & --- & $-4,1$ & $-3,4$ & --- & $-9,5$ & $-1,7$ \\
\hline
\end{tabular}




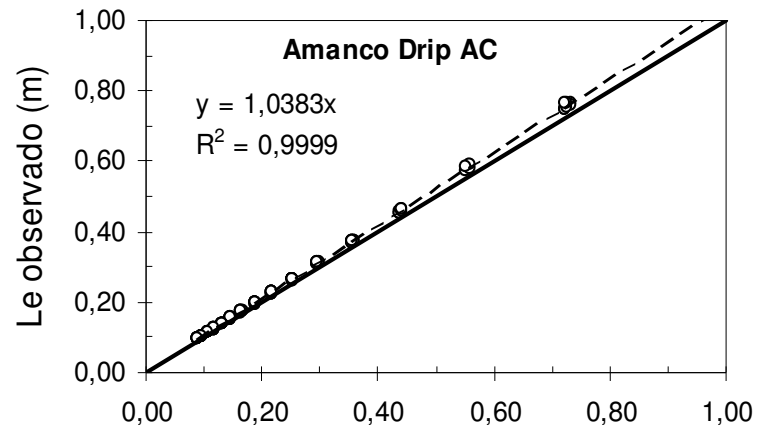

Le calculado $(\mathrm{m})$

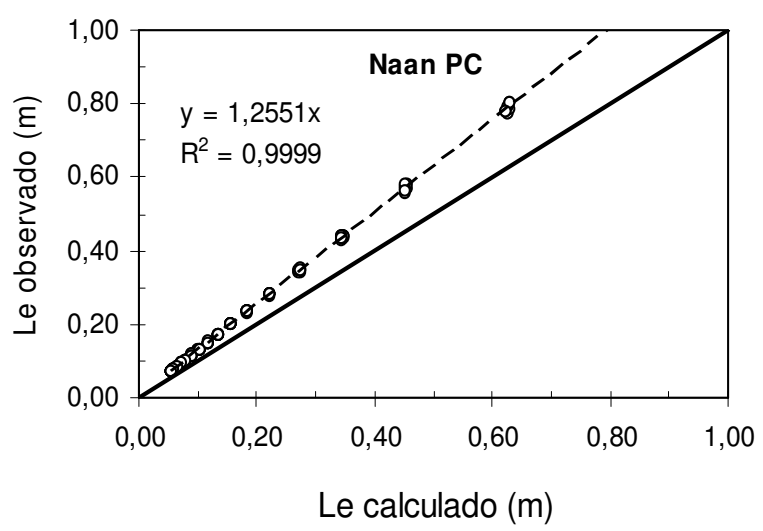

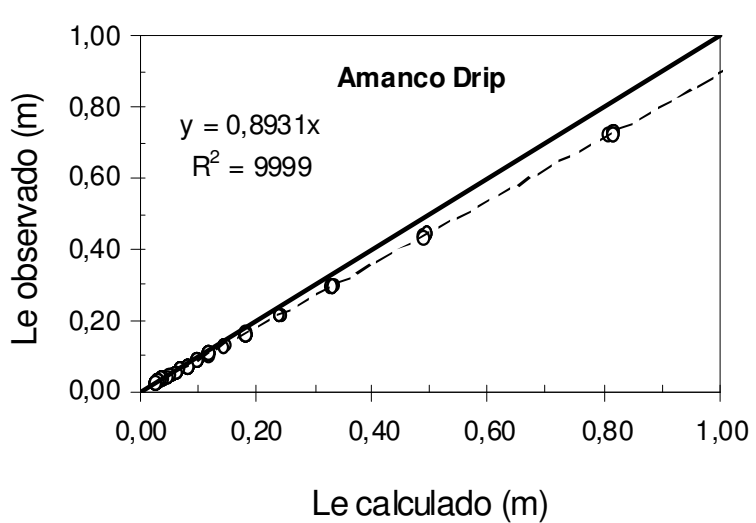

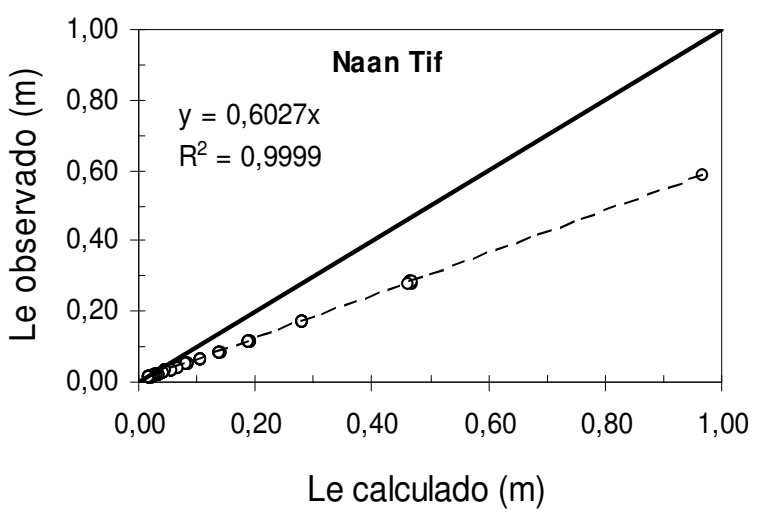

FIGURA 7. Comparação entre os comprimentos equivalentes obtidos em ensaios de laboratório e os comprimentos equivalentes calculados para cada emissor pela eq.(8). Comparison between the equivalent lengths obtained in laboratory and the equivalent lengths calculated for each emitter in eq. (8).

O cálculo da perda de carga localizada, utilizando-se do modelo potencial para estimar o valor de $\mathrm{K}$ (procedimento III), mostrou resultados pouco diferentes daqueles obtidos com o valor de $\mathrm{K}$ ajustado, com os dados observados em laboratório (procedimento II), o que resultou em pequenas variações na estimativa do comprimento máximo da linha lateral nesses dois procedimentos.

\section{CONCLUSÕES}

A consideração da perda de carga localizada é um critério relevante no dimensionamento de linhas laterais de gotejamento, especialmente quando se utilizam gotejadores autocompensados. A desconsideração da perda de carga localizada levou à superestimativa do comprimento máximo da linha lateral, de até $25,7 \%$ para os gotejadores autocompensados e de até 9,5\% para os gotejadores não autocompensados

O cálculo da perda de carga localizada, utilizando-se do modelo potencial para estimar o valor de $\mathrm{K}$, em função do índice de obstrução, mostrou bons resultados, comparáveis àqueles obtidos com o valor de $\mathrm{K}$ ajustado pelos dados observados em laboratório, o que resultou em pequenas variações na estimativa do comprimento máximo da linha lateral por esses dois procedimentos.

A perda de carga localizada, provocada pelos emissores, pode ser estimada satisfatoriamente pelo uso do coeficiente de carga cinética, calculado pelo modelo potencial, utilizando-se do índice de obstrução.

\section{AGRADECIMENTOS}

Ao Ministério da Ciência e Tecnologia (MCT), ao Conselho Nacional de Desenvolvimento Científico e Tecnológico (CNPq) e à Fundação de Amparo à Pesquisa do Estado de São Paulo 
(FAPESP), pelo apoio financeiro a esta pesquisa, através do Instituto Nacional de Ciência e Tecnologia em Engenharia da Irrigação (INCTEI).

\section{REFERÊNCIAS}

AL-MOUND, A.I. Significance of energy losses due to emitter connections in trickle irrigation lines. Journal of Agriculture Engineering Research, Silsoe, v.60, n.1, p.1-5, 1995.

ALVES, P.R.V.; PORTO, R. Coeficiente geométrico para estimativa da perda de carga localizada em linhas laterais de irrigação por gotejamento. Engenharia Agrícola, Jaboticabal, v.22, n.1, p.5159, 2002.

AZEVEDO NETTO, J.M. de; FERNANDEZ, M.F.; ARAÚJO, R. de; ITO, A.E. Manual de hidráulica. São Paulo: Edgard Blücher, 1998. 669 p.

BAGARELO, V.; FERRO, V.; PROVENZANO, G.; PUMO, D. Evaluating pressure losses in dripirrigation lines. Journal of Irrigation and Drainage Engineering, New York, v.123, n.1, p.1-7, 1997.

CARDOSO, G.G.G.; FRIZZONE, J.A. Perda de carga em linhas laterais de microirrigação. Revista Brasileira de Engenharia de Biossistemas, Campinas, v.1, n.2, p.147-162, 2007.

JUANA, L.; RODRIGUES-SINOBAS, L.; LOSADA, A. Determining minor head losses in drip irrigation laterals. I: Methodology. Journal of irrigation and Drainage Engineering, New York, v.128, n.6, p.376-384, 2002a.

JUANA, L.; RODRIGUES-SINOBAS, L.; LOSADA, A. Determining minor head losses in drip irrigation laterals. II: Experimental study and validation. Journal of Irrigation and Drainage Engineering, New York, v.128, n.6, p.385-396, 2002b.

KELLER, J.; KARMELI, D. Trickle irrigation design parameters. Transactions of the ASAE, St. Joseph, v.17, n.4, p.878-880, 1974.

PROVENZANO, G.; PUMO, D. Experimental analysis of local pressure losses for microirrigation laterals. Journal of Irrigation and Drainage Engineering, New York, v.130, n.4, p.318-324, 2004.

PROVENZANO, G.; PUMO, D.; DI DIO, P. Simplified procedure to evaluate head losses in drip irrigation lateral. Journal of Irrigation and Drainage Engineering, New York, v.131, n.6, p.525$532,2005$.

REDDY, K.Y. Evaluation of on-line trickle irrigation emitter barb losses. Journal of the Institution of Engineers- $A G$, Kharagpur, v.84, n.1, p.42-47, 2003.

RETTORE NETO, O.; FRIZZONE, J.A.; MIRANDA, J.H.; BOTREL, T.A. Perda de carga localizada em emissores não coaxiais integrados a tubos de polietileno. Engenharia Agrícola, Jaboticabal, v.29, n.1, p.28-39, 2009a.

RETTORE NETO, O.; MIRANDA, J.H.; FRIZZONE, J.A.; WORKMAN, S.R. Local head loss of non-coaxial emitters inserted in polyethylene pipe. Transactions of the ASABE, St. Joseph, v.52 n.3, p.729-738, 2009 b. 